\title{
Absence of collective effects in Heisenberg systems with localized magnetic moments
}

\author{
F. Illas and I. de P. R. Moreira \\ Departament de Química Física, Universitat de Barcelona, C/Martí i Franqués 1, 08028 Barcelona, Spain \\ C. de Graaf \\ Department of Chemical Physics, University of Groningen, Nijenborgh 4, Groningen 9747 AG, The Netherlands \\ O. Castell and J. Casanovas \\ Departament de Química, Universitat Rovira i Virgili, Pl. Imperial Tàrraco 1, 43005 Tarragona, Spain
}

(Received 28 May 1997)

\begin{abstract}
Existence of collective effects in magnetic coupling in ionic solids is studied by mapping spin eigenstates of the Heisenberg and exact nonrelativistic Hamiltonians on cluster models representing $\mathrm{KNiF}_{3}, \mathrm{~K}_{2} \mathrm{NiF}_{4}, \mathrm{NiO}_{\text {, }}$ and $\mathrm{La}_{2} \mathrm{CuO}_{4}$. Ab initio techniques are used to estimate the Heisenberg constant $J$. For clusters with two magnetic centers, the values obtained are about the same for models having more magnetic centers. The absence of collective effects in $J$ strongly suggests that magnetic interactions in this kind of ionic solids are genuinely local and entangle only the two magnetic centers involved. [S0163-1829(97)04634-1]
\end{abstract}

The proper and accurate description of magnetic interactions in systems with localized magnetic moments, i.e., ionic solids, is of importance not only from the point of view of basic knowledge but also to understand the electronic structure and magnetic behavior of superconductor parent compounds. ${ }^{1}$ The experimental and theoretical study of the magnetic coupling in ionic solids is often based on the use of the Heisenberg Hamiltonian which may be written as

$$
\hat{\mathcal{H}}=-\sum_{\langle i, j\rangle} J \hat{S}_{i} \hat{S}_{j}
$$

where the $\langle i, j\rangle$ symbol means that the summation is restricted to nearest-neighbor $i$ and $j$ magnetic centers with total spin moment $S$. The question addressed in this work concerns whether $J$ contains two-body interactions only or if it is better regarded as an effective two-body parameter containing collective effects from the whole solid. We must point out that existence of collective effects in $J$ is claimed mainly from intuition and has not been theoretically or experimentally proven.

Because ionic solids are extended systems, it is customary to investigate their electronic structure by using a solid-state approach, usually exploiting translational symmetry. Notice, that this approach cannot provide an answer to the above question because there is no way to separate the collective effects from the two-body interactions. From a purely $a b$ initio, or first-principles, point of view of the theory of the electronic structure, these solid-state approaches are often based on modifications ${ }^{2-6}$ of the local-density approach (LDA). Without these modifications the LDA fails to describe the antiferromagnetic order of many compounds such as $\mathrm{NiO}$ or $\mathrm{La}_{2} \mathrm{CuO}_{4}{ }^{7}$ More recently, the periodic HartreeFock, in its unrestricted or spin-polarized version, has been applied to a variety of antiferromagnetic systems with, on first sight, a rather good agreement with experiment. ${ }^{8,9}$ An alternative approach, different albeit complimentary, is one based on the use of cluster models. ${ }^{10-18}$ By construction, the cluster model permits one to investigate the importance of the collective effects. From a technical point of view, the main difference between periodic and local approaches lies in the way to estimate the instantaneous electron-electron interactions. Hence, while the former are constrained to the use of more or less approximate correlation (or exchangecorrelation) functionals, the latter permits the use of more accurate computational algorithms, usually based on configuration interaction expansions, that can be systematically improved with only the limitation of the computer resources. This local approach has permitted one to understand the basic mechanisms involved in magnetic coupling although a fully quantitative answer has not yet been possible. ${ }^{11,12,16-18}$

For three-dimensional (3D) magnetic systems such as $\mathrm{KNiF}_{3}$ and $\mathrm{NiO}$, the ab initio cluster model approach has been applied to the computation of the magnetic coupling constant, $J$, and been able to explain roughly $50 \%$ of the experimental value even after explicit inclusion of electronic correlation effects. ${ }^{11-14,17,18}$ However, for a two-dimensional (2D) magnetic system such as $\mathrm{La}_{2} \mathrm{CuO}_{4}$ the same approach permits one to obtain a much larger fraction $(\approx 80 \%)$ of the experimental value. ${ }^{16}$ In all cases, part of the remaining difference with respect to the experimental value has been attributed to the collective effects that might be included in $J$ [Eq. (1)]. Notice that for these systems the restriction on the summation in Eq. (1) to nearest neighbors only is well supported from experiments. ${ }^{19-26}$ In previous work, existence of collective effects was proposed from a cluster model containing more than two magnetic centers, but with a crude representation of the surroundings. ${ }^{11}$ Later, ab initio UHF periodic calculations on $\mathrm{KNiF}_{3}$ and $\mathrm{K}_{2} \mathrm{NiF}_{4}$ reported values of about $66 \%$ of experiment, considerably larger that those obtained by the cluster model approach, and what it is more surprising, without explicit consideration of electronic correlation effects. $^{8,9}$ These values appeared to provide further support for the existence of collective effects. However, in a very recent study Moreira and Illas have shown that, for $\mathrm{KNiF}_{3}$ and $\mathrm{K}_{2} \mathrm{NiF}_{4}$, the agreement between periodic and cluster 
UHF calculations was almost complete ${ }^{18}$ because the $J$ values calculated within the periodic approach reported in Refs. 8 and 9 used two different definitions of the Heisenberg Hamiltonian for theory and experiment, a constant factor of 2 being missing. The fact that, within the same $a b$ initio computational approach, cluster and periodic calculations conducts to the same calculated $J$ value for these two different $\mathrm{Ni}$ compounds is against the proposal that collective effects are responsible for a large fraction of the experimental magnetic coupling constant. Moreover, $a b$ initio cluster model calculations on $\mathrm{K}_{2} \mathrm{NiF}_{4}$ similar to those previously reported for $\mathrm{KNiF}_{3}$ yielded a similar description of $J$, only about 50\% of the experimental $J$ was recovered. This result is quite surprising since $\mathrm{K}_{2} \mathrm{NiF}_{4}$ is best described as a $2 \mathrm{D}$ magnetic system and, if the collective effect were to play an important role, one would expect a result closer to experiment as for $\mathrm{La}_{2} \mathrm{CuO}_{4} \cdot{ }^{10,15,16}$

In order to have conclusive answers on the role of collective effects we present here state-of-the-art $a b$ initio calculations for different cluster models representing $\mathrm{KNiF}_{3}$, $\mathrm{K}_{2} \mathrm{NiF}_{4}, \mathrm{NiO}$ (all with formal $\mathrm{Ni}^{2+}$ cations in a $d^{8}$ configuration) and $\mathrm{La}_{2} \mathrm{CuO}_{4}$ (with $\mathrm{Cu}^{2+}$ cations in a $d^{9}$ configuration). First, we will present results for models with two magnetic centers only. These results have been obtained at a very high level of theory, not available for larger cluster models. Next, we compare the $J$ values for two and many magnetic center cluster models. In this case special care has been taken that the ab initio methods applied permit an equivalent description for all cluster models. Now, $J$ contains either interactions from two magnetic centers only or explicitly includes effects arising from many magnetic center interactions. Comparison between the two sets of results will provide a direct answer about the role of collective effects.

For all systems, we define a local region which contains the nuclei and electrons that are explicitly treated and an outer, or embedding, region defined by an array of total ion potentials (TIP's) and point charges (PC's) placed at the bulk ion sites. TIP's account for the finite size of the cations closer to the local region and prevent artificial polarization of the ligand anions and PC's are added to have a good representation of the long-range Madelung potential. For $\mathrm{KNiF}_{3}$ and $\mathrm{K}_{2} \mathrm{NiF}_{4}$, the local regions are $\mathrm{Ni}_{2} \mathrm{~F}_{11}, \mathrm{Ni}_{3} \mathrm{~F}_{16}$, and $\mathrm{Ni}_{4} \mathrm{~F}_{20}$ cluster models where each magnetic center has its proper bulk coordination (Fig. 1). For $\mathrm{La}_{2} \mathrm{CuO}_{4}$, we use $\mathrm{Cu}_{2} \mathrm{O}_{11}$ and $\mathrm{Cu}_{2} \mathrm{O}_{7}$ for the two magnetic center models and $\mathrm{Cu}_{3} \mathrm{O}_{10}$ and $\mathrm{Cu}_{5} \mathrm{O}_{16}$ models for the many magnetic center models. Notice that $\mathrm{Cu}_{2} \mathrm{O}_{7}, \mathrm{Cu}_{3} \mathrm{O}_{10}$, and $\mathrm{Cu}_{5} \mathrm{O}_{16}$ contain inplane oxygen atoms only; as shown recently apical oxygen makes only a tiny contribution to $J .{ }^{16}$ Finally, the NiO, the local regions are simply $\mathrm{Ni}_{2} \mathrm{O}_{11}$ and $\mathrm{Ni}_{3} \mathrm{O}_{16}$.

Once the cluster models are defined, we obtain $J$ by a suitable mapping of the eigenstates of exact nonrelativistic and Heisenberg Hamiltonians. ${ }^{11-18}$ For the exact nonrelativistic Hamiltonian we obtain reasonable estimates of the proper eigenstates and eigenvalues using a multireference zeroth-order wave function and by explicitly accounting for external correlation effects with difference dedicated CI (Ref. 27) (DDCI), and/or multireference second-order perturbation theory ${ }^{28}$ for the pertinent spin states. In these calculations, the reference space is defined by the eigenfunctions of a complete active space configuration interaction (CASCI)

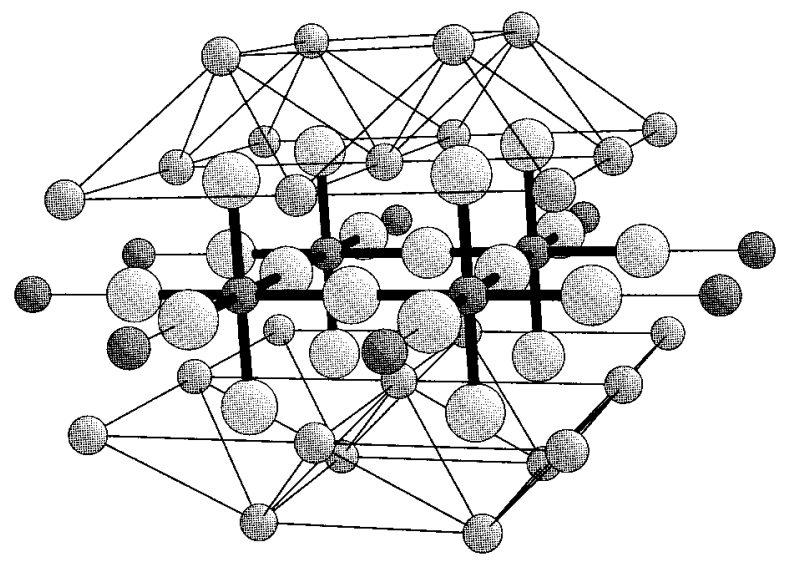

FIG. 1. Schematic representation of the $\mathrm{Ni}_{4} \mathrm{~F}_{20}$ cluster model used to represent $\mathrm{K}_{2} \mathrm{NiF}_{4}$. Also shown are the total ion potentials for the nearest $\mathrm{K}^{+}$and $\mathrm{Ni}^{2+}$ cations surrounding the $\mathrm{Ni}_{4} \mathrm{~F}_{20}$ cluster. Thick lines link cluster atoms while thin lines link cluster atoms to TIP's; small dark spheres represent $\mathrm{Ni}^{2+}$, small light spheres $\mathrm{K}^{+}$, and large spheres $\mathrm{F}^{-}$anions.

expansion. The active space consists of the cation open shells, or magnetic orbitals, with their corresponding electrons. Hence, for $\mathrm{Ni}$ compounds, the magnetic orbitals are $d_{z^{2}}$ and $d_{x^{2}-y^{2}}$ and, for both $\mathrm{Ni}_{4} \mathrm{~F}_{20}$ clusters, the CAS contains 8 electrons in 8 orbitals whereas for both $\mathrm{Ni}_{3} \mathrm{~F}_{16}$ and $\mathrm{Ni}_{3} \mathrm{O}_{16}$ it contains 6 electrons in 6 orbitals. Likewise, in the case of $\mathrm{La}_{2} \mathrm{CuO}_{4}$ there is one $d_{x^{2}-y^{2}}$ magnetic orbital per center and, for $\mathrm{Cu}_{5} \mathrm{O}_{16}$, the CAS has 5 electrons in 5 orbitals. The CASCI eigenfunctions are expansions of Slater determinants built up of molecular orbitals (MO's) which in turn are expressed as a linear combination of Gaussian-type atomic basis functions. The MO's are obtained from a spinrestricted open-shell Hartree-Fock calculation on the highest spin state. The external correlation, out of the CAS, is accounted for by exploiting the ideas of quasidegenerate perturbation theory (QDPT) and taking the CAS as model space. In this case the off-diagonal elements of the secondorder effective Hamiltonian, $\hat{\mathcal{H}}_{I, J}^{\text {eff(2) }}$, take the following form:

$$
\hat{\mathcal{H}}_{I, J}^{\mathrm{eff}(2)}=\sum_{I, J \in \mathrm{CAS}} \sum_{K \notin \mathrm{CAS}} \frac{\langle I|\hat{\mathscr{H}}| K\rangle\langle K|\hat{\mathscr{H}}| J\rangle}{E_{K}^{0}-E_{J}^{0}}
$$

and the $|K\rangle$ determinants are single and double excitations from the CAS determinants. Depending on the number of differences in holes and particles with respect to those in the CAS, the $|K\rangle$ determinants may have two, three, or four degrees of freedom. ${ }^{27}$ In the DDCI, the $|K\rangle$ determinants with two degrees of freedom are treated up to infinite order. This is because these determinants are the ones with the largest contribution to the energy difference between the eigenvalues of the second-order effective Hamiltonian. ${ }^{27}$ In the present work, for the clusters with two magnetic centers, the external correlation is included in two parts. The determinants with two degrees of freedom are treated up to infinite order through DDCI and the rest of the terms in Eq. (2) by second-order perturbation theory. These calculations are carried out at the all-electron level using large basis sets (series $A)$. For the larger clusters, the external correlation is always included up to second order. Moreover, the core electrons are 
TABLE I. Calculated and experimental $J$ values for different compounds extracted from different cluster models. Series A refers to the all electron calculations on the two magnetic center models whereas series $B$ corresponds to the pseudopotential description of two and many magnetic center models.

\begin{tabular}{lcccr}
\hline \hline Compound & Model & & $J(\mathrm{meV})$ & \\
& & Series A & & Series B \\
\hline $\mathrm{KNiF}_{3}$ & $\mathrm{Ni}_{2} \mathrm{~F}_{11}$ & -6.1 & & -3.8 \\
& $\mathrm{Ni}_{3} \mathrm{~F}_{16}$ & & & -3.9 \\
& $\mathrm{Ni}_{4} \mathrm{~F}_{20}$ & & & -4.0 \\
Expt (Refs. 19-21) & & & -7.7 & \\
$\mathrm{~K}_{2} \mathrm{NiF}_{4}$ & $\mathrm{Ni}_{2} \mathrm{~F}_{11}$ & -6.6 & & -4.1 \\
& $\mathrm{Ni}_{3} \mathrm{~F}_{16}$ & & & -4.4 \\
& $\mathrm{Ni}_{4} \mathrm{~F}_{20}$ & & & -4.3 \\
Expt (Refs. 19-21) & & & -8.6 & \\
La $\mathrm{CuO}_{4}$ & $\mathrm{Cu}_{2} \mathrm{O}_{11}$ & -100.1 & & -88.4 \\
& $\mathrm{Cu}_{2} \mathrm{O}_{7}$ & & & -92.4 \\
& $\mathrm{Cu}_{3} \mathrm{O}_{15}$ & & & -96.6 \\
& $\mathrm{Cu}_{5} \mathrm{O}_{20}$ & & & -95.1 \\
Expt (Refs. 22-25) & & & -120.0 & \\
NiO & $\mathrm{Ni}_{2} \mathrm{O}_{11}$ & -10.6 & & -7.8 \\
& $\mathrm{Ni}_{3} \mathrm{O}_{16}$ & & & -8.1 \\
Expt. (Ref. 26) & & & -19.8 & \\
\hline \hline
\end{tabular}

replaced by a suitable pseudopotential (series $B$ ). Results for the two magnetic center model have also been carried out at this level for comparison purposes. Further technical details on basis set, pseudopotentials, and embedding can be found in Refs. 12, 14, and 16-18.

In Table I we present a brief summary of results for the different systems described above. First, we discuss the $a b$ initio all-electron calculations for the cluster models with two magnetic centers (series $A$ ). In all cases, agreement with experiment is quite impressive. This is an important point given the very small energy differences involved and show the level of accuracy of the $a b$ initio methods used in the present approach. Now, we come to the key point of this work. This concerns the origin of the difference between calculated and experimental results. It may be due to limitations of the computational approach: the requirement to include higher orders in the treatment of external correlation and the need to enlarge the active space, or to the limitations on the cluster model approach: the necessity to include more magnetic centers. At present it is hardly possible to go beyond the level of accuracy given by series $A$ but we may investigate the limitations of this cluster model approach by considering the results for many magnetic center models. For the four materials and the different clusters, the general trend is the same; namely, that the calculated value of $J$ (series $B$ ) is found to be almost independent of the number of magnetic centers explicitly included in the $a b$ initio computational model. Given the fact that the systems chosen to carry out this study are very different, this is a highly surprising result. In fact, it holds for the $\mathrm{KNiF}_{3}$ and $\mathrm{K}_{2} \mathrm{NiF}_{4}$ perovskites, 3D and 2D magnetic systems, but where the proper coordination of each cation is essential to describe the magnetic interaction. This is because the magnetic orbitals $d_{z^{2}}$ and $d_{x^{2}-y^{2}}$ are both directed straight towards the anions. Thus, the presence of the ligands influences the spatial extent of the magnetic orbitals and hence the magnetic moment and the magnetic coupling constant. This strong influence of the ligands permits one to understand why a simpler $\mathrm{Ni}_{4} \mathrm{~F}_{4}$ cluster model, where the cations did not have the proper environment, led to a $J$ value larger than that for a $\mathrm{Ni}_{2} \mathrm{~F}$ model and seemed to provide an argument for the existence of collective effects. Clearly this was an arbitrary, unexpected, cluster artifact. We must point out that, although not given in the table, the results corresponding to the CASCI wave function (which include essentially the terms arising from Anderson superexchange mechanism) exhibit the same trend. The calculated $J$ values are, of course, smaller but they do not evidence any dependence with respect to the number of magnetic centers.

The conclusion of this work can be summarized in a very concise way. Upon consideration of the correct mapping between spin eigenstates of the Heisenberg and using a reliable $a b$ initio representation of those of the exact nonrelativistic Hamiltonian, for cluster models with two or more magnetic centers it is possible to obtain a reasonable estimate of the magnetic coupling constant $J$ and to investigate the possible existence of collective effects. Surprisingly enough, the $J$ value obtained from clusters with two magnetic centers is about the same as obtained when more magnetic centers are explicitly included in the model. This is found to be the case for four different materials with fairly different physical and chemical properties. Previous attempts to proclaim the existence of collective effects have been found to arise from cluster artifacts or from an improper comparison between calculated and experimental results (see Ref. 18). The absence of collective effects in the magnetic coupling constant found for $\mathrm{KNiF}_{3}, \mathrm{~K}_{2} \mathrm{NiF}_{4}, \mathrm{NiO}$, and $\mathrm{La}_{2} \mathrm{CuO}_{4}$ strongly suggests that the magnetic interactions in this kind of narrow band systems are genuine local and entangle only the two magnetic centers involved.

This research was supported by the Human Capital and Mobility Programme, Access to Large Installations, under Contract No. CHGE-CT92-0009 “Access to supercomputing facilities for European researchers"' established between the European Community and CESCA/CEPBA. We also acknowledge financial support from the Spanish "Ministerio de Educación' under Project Nos. DGICYT PB95-0847C02-01 and PB95-0847-C02-02 and partial support from the " 'Direcció General de Recerca de la Generalitat de Catalunya" under Project Nos. 1995SGR-00048 and 1995SGR00426. Finally, the authors wish to thank the "Centre de Supercomputació de Catalunya,' CESCA, for helping with the calculations. 
${ }^{1}$ J. G. Bednorz and K. A. Müller, Earlier and Recent Aspects of Superconductivity (Springer, New York, 1990).

${ }^{2}$ A. Svane, Phys. Rev. Lett. 68, 1900 (1992).

${ }^{3}$ A. Svane and O. Gunnarsson, Phys. Rev. Lett. 65, 1148 (1990).

${ }^{4}$ M. T. Czyzyk and G. A. Sawatzky, Phys. Rev. B 49, 14211 (1994).

${ }^{5}$ V. I. Anisimov, M. A. Korotin, J. A. Zaanen, and O. K. Andersen, Phys. Rev. Lett. 68, 345 (1992).

${ }^{6}$ P. Wei and Z. Q. Qi, Phys. Rev. B 49, 12519 (1994).

${ }^{7}$ W. E. Pickett, Rev. Mod. Phys. 61, 433 (1989).

${ }^{8}$ J. M. Ricart, R. Dovesi, C. Roetti, and V. R. Saunders, Phys. Rev. B 52, 2381 (1995).

${ }^{9}$ R. Dovesi, J. M. Ricart, V. R. Saunders, and R. Orlando, J. Phys.: Condens. Matter 7, 7997 (1995).

${ }^{10}$ R. L. Martin, J. Chem. Phys. 98, 8691 (1993).

${ }^{11}$ F. Illas, J. Casanovas, M. A. Garcia-Bach, R. Caballol, and O. Castell, Phys. Rev. Lett. 71, 3549 (1993).

${ }^{12}$ J. Casanovas and F. Illas, J. Chem. Phys. 100, 8257 (1994).

${ }^{13}$ J. Casanovas and F. Illas, J. Chem. Phys. 101, 7683 (1994).

${ }^{14} \mathrm{~J}$. Casanovas, J. Rubio, and F. Illas, in New Challenges in Computational Quantum Chemistry, edited by R. Broer, P. J. C. Aerts, and P. S. Bagus (University of Groningen, Groningen,
1994), pp. 214-226.

${ }^{15}$ A. B. Van Oosten, R. Broer, and W. C. Nieuwpoort, Int. J. Quantum Chem. Quantum Biol. Symp. 29, 241 (1995).

${ }^{16}$ J. Casanovas, J. Rubio, and F. Illas, Phys. Rev. B 53, 945 (1996).

${ }^{17}$ C. de Graaf, F. Illas, R. Broer, and W. C. Nieuwpoort, J. Chem. Phys. 106, 3287 (1997).

${ }^{18}$ I. de P. R. Moreira and F. Illas, Phys. Rev. B 55, 4129 (1997).

${ }^{19}$ M. E. Lines, Phys. Rev. 164, 736 (1967).

${ }^{20}$ L. J. de Jongh and R. Miedema, Adv. Phys. 23, 1 (1974).

${ }^{21}$ L. J. de Jongh and R. Block, Physica B 79, 568 (1975).

${ }^{22}$ G. Aeppli et al., Phys. Rev. Lett. 62, 2052 (1989).

${ }^{23}$ Y. Endoh et al., Phys. Rev. B 37, 7443 (1988).

${ }^{24}$ K. Yamada et al., Phys. Rev. B 40, 4557 (1989).

${ }^{25}$ R. R. P. Singh, P. A. Fleury, K. B. Lyons, and P. E. Sulewski, Phys. Rev. Lett. 62, 2736 (1989).

${ }^{26}$ M. J. Masey, N. H. Chen, J. W. Allen, and R. Merlin, Phys. Rev. B 42, 8776 (1990).

${ }^{27}$ J. Miralles, O. Castell, R. Caballol, and J. P. Malrieu, Chem. Phys. 172, 33 (1993).

${ }^{28}$ B. Huron, P. Rancurel, and J. P. Malrieu, J. Chem. Phys. 58, 5745 (1973); S. Evangelisti, J. P. Daudey, and J. P. Malrieu, Chem. Phys. Lett. 75, 91 (1983). 